\title{
Innovative Radiating Systems for Train Localization in Interference Conditions
}

\author{
C. Vegni, ${ }^{1}$ M. Tosti, ${ }^{2}$ and A. M. Vegni $^{3}$ \\ ${ }^{1}$ Thales Alenia Space Italia, Navigation \& Integrated Communication Directorate, Via Saccomuro 24, 00131 Rome, Italy \\ ${ }^{2}$ NEXT, Ingegneria dei Sistemi, Via Andrea Noale 345/b, 00155 Rome, Italy \\ ${ }^{3}$ University Roma Tre, Department of Engineering, Via Vito Volterra 62, 00146 Rome, Italy
}

Correspondence should be addressed to C. Vegni; claudio.vegni@thalesaleniaspace.com

Received 5 December 2012; Revised 21 February 2013; Accepted 3 June 2013

Academic Editor: Renato Cicchetti

Copyright (C) 2013 C. Vegni et al. This is an open access article distributed under the Creative Commons Attribution License, which permits unrestricted use, distribution, and reproduction in any medium, provided the original work is properly cited.

\begin{abstract}
The design of innovative radiating systems based on the metamaterial technology for GNSS (Global Navigation Satellite System) applications in radio frequency (RF) interference conditions is proposed. To this aim, firstly two typical adaptive array techniques (i.e., nulling and beam-forming) are discussed and tradeed off. Secondly, FRPA (Fixed Radiation Pattern Antenna) and CRPA (Controlled Radiation Pattern Antenna) phased array configurations of miniaturized patch antennas are studied by means of electromagnetic commercial tools and phased array optimization algorithms. This process leads to the identification of a phased array design. Benefits and drawbacks for GNSS applications are highlighted. Finally, the design of the phased array is applied to a GNSS user receiver in a navigation realistic environment. Simulation results are obtained in a realistic scenario for railway applications, comprising of a GNSS satellite constellation, a GNSS user receiver (i.e., on-board train equipment) running along a track in Western Australia, and a constellation of interfering satellites. Navigation service performances (i.e., user location accuracy and service availability) are computed taking into account the adaptive array radiation pattern in two different modes (i.e., FRPA or CRPA) and band-limited white noise interference.
\end{abstract}

\section{Introduction}

Concern about GPS (Global Positioning System) vulnerability has received a great deal of attention recently $[1,2]$. GNSS receivers are highly susceptible to interference caused by jamming and spoofing. Historically, signal jamming and the design have been primarily considered as a military problem. Now, signal interference threatens all the GNSS receivers, military and civilian.

GPS signal typical level on Earth surface is around $-163 \mathrm{~dB} \mathrm{~W}$, that is, below the noise floor of the receiver (i.e., $N=K \cdot T_{\text {SYS }} \cdot B$, e.g., $-228.6+27+63 \mathrm{~dB}-\mathrm{Hz}=-138 \mathrm{~dB}$ for $20 \mathrm{MHz}$ bandwidth L band signal).

The reason why GPS signals can survive to degradation effects like interference is due to the spread-spectrum nature of the transmission, allowing receivers to correlate the satellite signal out from below the background noise [2,3]. However, each receiver exhibits a limitation to the amount of non-GNSS interference it can cope with, whilst still acquiring or tracking the desired signals. In other words, each receiver has a maximum Jammer-to-Signal ratio (i.e., J/S) that is able to tolerate.

Recent studies $[1,2]$ demonstrated that a $10 \mathrm{~mW}$ jammer at a distance less than $10 \mathrm{~km}$ can seriously disturb the acquisition process of a GPS receiver. Jamming is not the only form of GNSS interference; spoofing is a more recent one. Spoofing (i.e., data level interference) can be considered as a more dangerous form of interference than jamming, because it is not obvious that the GNSS receiver is being spoofed, while jamming constitutes a denial of service and it is easily detectable.

In this paper a band-limited white noise interference is considered. When the interference has flat spectrum centred in $f$ frequency and extending in the range $\left(f_{i}-\beta_{i} / 2\right) \leq f \leq$ $\left(f_{i}+\beta_{i} / 2\right)$, its spectrum is expressed as follows [2]:

$$
S_{i}(f)= \begin{cases}\frac{1}{\beta_{i}}, & \left(f_{i}-\frac{\beta_{i}}{2}\right) \leq f \leq\left(f_{i}+\frac{\beta_{i}}{2}\right), \\ 0, & \text { elsewhere. }\end{cases}
$$




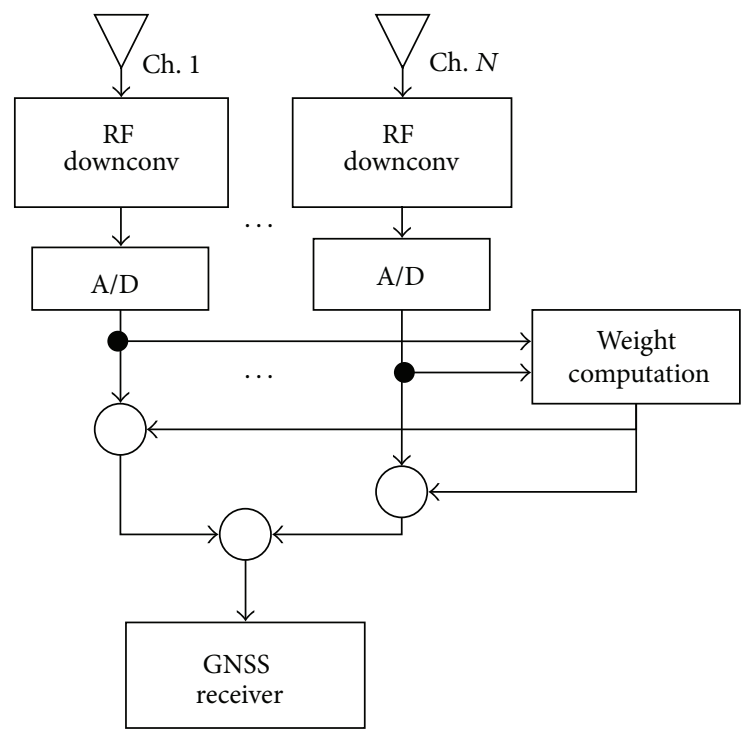

FIGURE 1: Single-output nulling antenna block diagram.

RF interference and multipath effects can be mitigated or eliminated by an appropriate GNSS antenna and receiver design [1]. In particular, we focus on antenna solutions as adaptive arrays. They are considered the single most powerful antijamming tool in the GNSS systems engineer's toolkit. They provide jamming rejection from 15 to $90 \mathrm{~dB}$ depending on the specific architecture used. Their main drawback is that they require an array of antenna elements, each spaced about ten centimetres (center-to-center) in S band, and thus their physical envelope cannot meet the antenna room constraints.

Therefore, the paper will be organized according to three following steps.

(1) Adaptive array typology trade-off: two typical techniques (i) nulling and (ii) beam-forming are discussed and compared in Section 2.

(2) Adaptive array antenna design: in Section 3 the RF design of a phased array antenna is presented in terms of array geometric layout and single radiator element in metamaterial technology.

(3) GNSS study case: a realistic navigation case is investigated in Section 4. Specifically, the train localization problem by GPS satellites is studied in conditions of RF interfering satellites.

FRPA and CRPA solutions are considered, and for each one GPS service performances (i.e., train accuracy and service availability) are computed. In other words, the RF design is validated in terms of GNSS system performances in a realistic scenario. Conclusions are finally drawn.

\section{Adaptive Array Typology Trade-Off}

Two types of adaptive array antenna are used with GNSS receivers, that is, (i) single-output nulling (Figure 1) antennas, and (ii) multiple-output beam-steering (Figure 2) antennas [4].

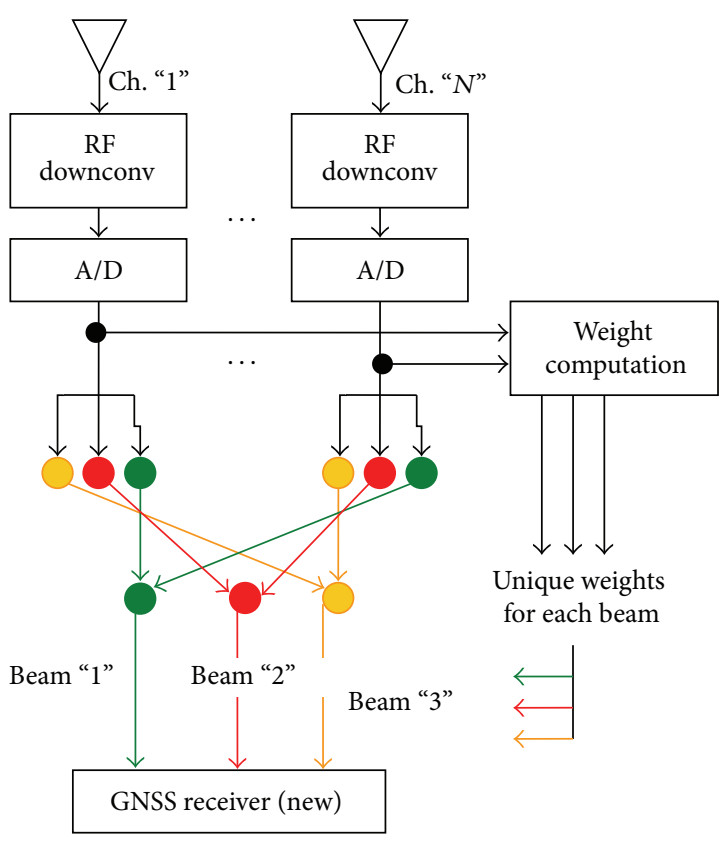

FIGURE 2: Multiple-output beam-steering antenna block diagram.

Most of deployed systems are single-output adaptive nulling antennas that operate as an antijamming appliqué. In this way the GNSS receiver does not need to know what type of antenna is connected to, that is, FRPA or CRPA.

Some development systems [6] tend to emphasize multiple-output beam-steering antennas because of their better performance. However, in order to handle the multipleoutput channels, a new receiver is required too. The trend is to integrate the array processing with the GNSS receiver in a single unit.

On the other side, a single-output nulling antenna uses a common set of weights for all GNSS signals. The objective is to minimize the output power subject to the constraint that one element is turned on. Remaining channels have their output phase and amplitude dynamically adjusted, so as to cancel out jammers in the summation process. The result consists in very sharp spatial nulls in the directions of the jammers but, because the GNSS signal direction of arrival is not taken into account, a significant possibility arises for low gain in some signal directions.

However, in GNSS applications, the antenna has to receive navigation signal from as many satellites as possible.

On the other side, GNSS antenna is requested to reject as many interfering satellites as possible along single or multiple directions.

In principle, an $N$-element array can independently steer $N-1$ spatial nulls in the direction of jammers. Moreover, in the multiple-output beam-forming array configuration, a unique set of weights is generated and optimized for each signal. The weight information process takes into account the desired signal direction of arrival, and, in absence of jamming, it will phase the input channels so they coherently add together to create a beam in the direction of the satellite. 
TABLE 1: Adaptive antenna nulling versus beam-forming techniques.

\begin{tabular}{lcc}
\hline$N$-elements antenna feature & Nulling & Beam-forming \\
\hline Robustness to interference & Low (low gain along not IF directions) & Medium (adequate gain roll-off shown) \\
Maximum number of spatial nulls & $N-1$ & $N-1$ \\
Null depth & $<-20 \mathrm{~dB}$ & $<-20 \mathrm{~dB}$ \\
Directional beam capability & No & Yes \\
Impact on accuracy measurement & Not compatible with high precision & More than $100^{\circ}$ on carrier phase meas.; \\
& applications & 1 meter on code phase meas. \\
\hline
\end{tabular}

In addition, to enable an adaptive antenna array to steer minimal antenna gain towards jammers, beam-forming simultaneously ensures that the antenna steers maximum gain towards GNSS satellites.

In other words, instead of minimizing the interferenceto-noise ratio (INR), the aim is to maximize the signal-tointerference-plus-noise ratio (SINR).

Like the nuller, the beam-former can independently steer $N-1$ spatial nulls.

Both the nuller and the beam-former perform much better (i.e., $>20 \mathrm{~dB}$ ) against narrow-band jammers.

The advantages of nulling and beam-forming antennas can be reported as follows.

(i) Nulling solution appears less complex than beamformer one.

(ii) Nullers synthetize sympathetic nulls in directions other than those of jammers. Sometimes, this happens in the direction of a desired signal and the signal is lost. It corresponds to a decrease of the number of visible satellites and consequently to a degradation of GNSS performance parameters (e.g., accuracy, continuity).

(iii) Beam-formers provide a broad-beam in the direction of the desired signal and lower gain in other directions. Multipath arriving from low gain directions is more strongly attenuated and has less ability to corrupt code and carrier phase observables.

(iv) Nullers do not provide this performance gain as they do not seek to generate directional beam.

In general, adaptive arrays improve signal-to-noise ratio in presence of jamming and thus permit signal tracking in environments that otherwise would lack code and carrier phase observables (lack of pseudorange measurement). However, this benefit is not without cost: adaptive arrays can bias code and carrier phase observables. The array antenna is a spatial filter, and its distortion effects are also direction dependent. The distortion is unique for each desired signal and does not necessarily common-mode out in subsequent processing.

In absence of jamming, the biases are fairly benign, while in jamming conditions the biases can become large and sustained.

Beam-formers can show 100-degree carrier phase biases and upwards of 1-meter code phase biases.

Nullers do even worse. For high precision systems, these errors can be a significant component of the overall error budget. Table 1 summarizes the previous considerations.
In this paper, the synthesis algorithm MVDR (Minimum Variance Distortionless Response) is applied [4, 6]. It falls into the category of beam-forming adaptive array. This algorithm allows the synthesis of up to $N-1$ nulls and the pointing of the main beam along one single direction. The considered direction is the broad-side one $\left(\theta=90^{\circ}\right.$ with respect to horizon).

In case interfering direction is close to or coincides with the broad-side one, the algorithm shall show convergence problems.

However, this satellite geometric configuration is highly unlikely. In that case the antenna beam shall point to a direction computed as the mean value among all the interfering directions.

\section{Adaptive Array Antenna Design}

In this section, firstly, we show the basic concepts to be followed to design a GNSS antenna. Secondly, the phased array element innovative design is illustrated by VSWR, axial ratio (AR), and radiation pattern curves. Finally, the adaptive beam-forming phased array design is shown, in terms of array layout and radiation patterns.

3.1. GNSS Antenna Guidelines. The guidelines consider four typical specific antenna parameters, that is, (i) pattern, (ii) roll-off, (iii) polarization purity, and (iv) phase centre.

(i) Antenna pattern: the GNSS antenna has to receive all the navigation signals transmitted by the GNSS satellites in visibility. Ideally, it should show an omnidirectional radiation pattern. The GPS receiver operates best with only a small difference in power among the signals from the various satellites, and, ideally, the antenna covers the entire hemisphere with no variation in gain. This has to do with potential cross-correlation problems in the receiver and the fact that excessive gain roll-off may cause signals from satellites at low elevations to drop well below the noise floor of the receiver. On the other hand, optimization for multipath rejection and antenna noise temperature require some gain roll-off.

(ii) Antenna roll-off: antenna should show perfect hemispherical radiation pattern. However, such an antenna cannot be practically built, and real-world GNSS antennas experience a gain roll-off of 10 to $20 \mathrm{~dB}$ from broad-side to the horizon. 
TABLE 2: GNSS antenna design guidelines.

\begin{tabular}{ll}
\hline Antenna parameter & Guidelines for GNSS applications \\
\hline Radiation pattern & Hemispherical \\
Roll-off & $\begin{array}{l}11-13 \mathrm{~dB} \text { (from broad-side to } 10^{\circ} \text { elevation } \\
\text { direction) }\end{array}$ \\
Polarization purity & $\begin{array}{l}\text { (i) } 1 \mathrm{~dB} \text { at broad-side } \\
\text { (ii) }<6 \mathrm{~dB} \text { at } 10^{\circ} \text { elevation }\end{array}$ \\
Phase centre & (i) Stable versus frequency \\
& (ii) Stable versus $\mathrm{Az}, \mathrm{El}$ angles \\
\hline
\end{tabular}

(iii) Antenna polarization purity: a right hand circular polarization (RHCP) antenna will pick up some left hand circular polarization (LHCP) energy. For highend (geodetic/choke ring) GNSS antennas, the typical AR in broad-side should be around $1 \mathrm{~dB}$. AR increases toward the lower elevations (less than 3 to $6 \mathrm{~dB}$ at $10^{\circ}$ elevation for a high-performance antenna). Maintaining a good AR (i.e., 1-2 dB) over the entire hemisphere and at all the frequencies is a challenge for the antenna RF designer. Good polarization purity represents a valid rejection to multipath/interference effect. In fact, reflected signals are LHCP (single reflection). Consequently, multipath signals from reflections against vertical objects such as buildings can be deeply attenuated or even suppressed.

(iv) Antenna phase centre: in GNSS applications, the user position is relative to the phase centre of the GNSS receiving antenna. The phase centre is the point in space from which all the rays appear to emanate (or converge on) the antenna. Put in another way, it is the point where the fields from all the incident rays appear to add up in phase. Determining the phase centre is important in GNSS systems, particularly when millimetre positioning resolution is desired. Ideally, the phase centre is a single point in space for all directions at all frequencies. A real-world antenna often will possess multiple phase centre points or a phase centre that appears "smeared out" as frequency and viewing angle vary. For well-designed high-end (geodetic/choke ring) GNSS antennas, phase centre variations in azimuth are small and in the order of a couple of millimetres. The vertical phase offsets are typically $10 \mathrm{~mm}$ or less.

The above-discussed guidelines can be summarized in Table 2 .

3.2. Element Design. The single radiator configuration is shown in the top view (Figure 3), in the cross-view (Figure 4), and in $3 \mathrm{D}$ view (Figure 5). This is a dual probe miniaturized monolayer patch in L1 band, at $1.55 \mathrm{GHz}$ central frequency. The circular polarization is obtained by two inputs (probes). Each probe is electromagnetically coupled to the radiating patch by a parasitic disk. Moreover, four mushrooms (depicted in yellow in Figure 3) are introduced to reduce the envelope of the squared ring. In particular, mushrooms are folded (dotted lines) in order to improve capacitive effects.

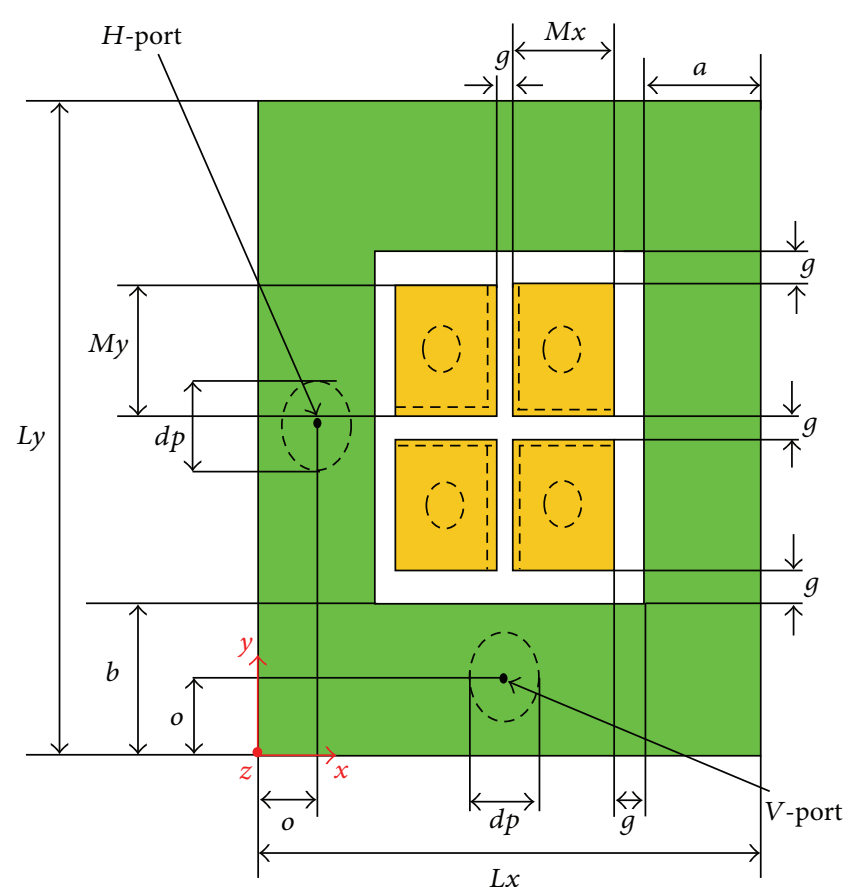

Figure 3: Single element-top view.

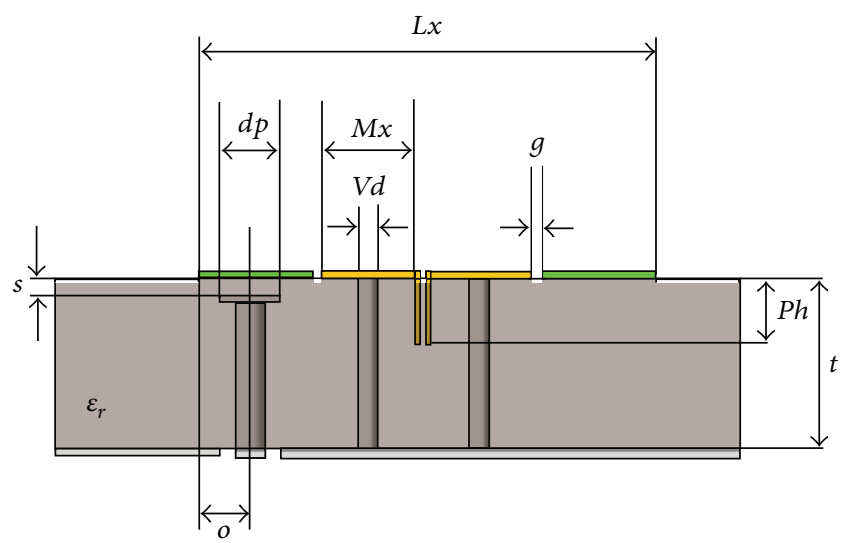

FIgURe 4: Single element-cross view (V-port not shown).

Balanced feeds tend to have superior scanning performance as the higher order modes are naturally suppressed by the symmetrical feeding arrangement. Moreover, polarization purity is improved using two inputs instead of a single port. A dual-probe interconnect is chosen as a result of these performance advantages.

The miniaturized monolayer patch can be easily obtained by using the metamaterial technology. It is well known that this technology allows several advantages in the realization of antennas, namely, the printed ones. One of these advantages (miniaturization) is obtained by using "metamaterial-based" antennas or "metamaterial-inspired" ones.

The difference among "metamaterial-based" antennas and "metamaterial-inspired" ones stands either using unconventional dielectric slabs (for "metamaterial-based" 


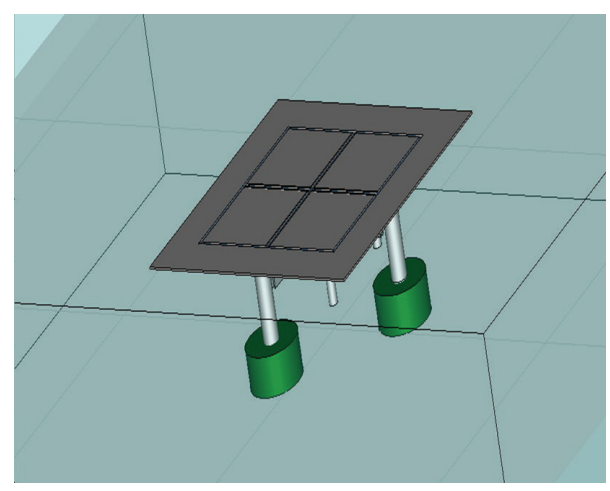

Figure 5: Single element 3D view.

antennas) or parasitic elements/cuts nearby/inside the radiating patch (for "metamaterial-inspired" antennas) [7-12].

This means that major dimension of metamaterial antenna elements used in array configuration is smaller than usual ones (from $\lambda_{g} / 2$ to $\lambda_{g} / 20$ or more, corresponding to an area reduction of $90 \%$ or more). Consequently, the dimensions of the whole antenna (e.g., the array) can be drastically reduced $[13,14]$.

The patch ground-plane separation is about $5 \mathrm{~mm}$, and the underlying substrate permittivity is 2.2 . The envelope area is $3.38 \mathrm{~cm}^{2}$ (i.e., $1.84 \times 1.84 \mathrm{~cm} \approx 3.38$ ). It is noted that, at the same working frequency $(1.55 \mathrm{GHz})$ in case of standard monolayer (i.e., $\varepsilon_{r}=2.2$ ) patch (typical $\lambda_{g} / 2$ ), the single element envelope would be $6.41 \times 6.41=41.08 \mathrm{~cm}^{2}$. Metamaterial radiator technology area is the $3.38 / 41.08=$ $8.22 \%$ of a standard monolayer patch.

A Cartesian reference system adopted to define the field quantities is introduced in Figure 3. Its origin is coincident to the left-side angle of the squared ring as shown in the figure.

Table 3 reports the single element physical parameters (distances expressed in $\mathrm{mm}$ ).

Finally, the $-15 \mathrm{~dB}$ return loss (Figure 6) bandwidth is 25.2 $\mathrm{MHz}(1.6 \%)$.

On the other side, the reduction of the antenna aperture generally corresponds to a decrease of the single element antenna gain. This effect has been taken into account in the design of the phased array antenna. The radiation pattern is shown in Figure 7, where it can be noted the peak gain around $5.7 \mathrm{dBi}$.

The axial ratio value is about $2 \mathrm{~dB}$ and shows a flat trend (Figure 8). It is in line with the guidelines described in the previous paragraph which are low $\mathrm{AR}$ values for low elevation angles.

3.3. Array Layout Optimization. The antenna aperture is a design driver. The layout definition process is addressed considering the following aspects.

(i) The envelope diameter shall be compatible to train roof accommodation, that is, $80 \mathrm{~cm}$ (goal) and $30 \mathrm{~cm}$ (nice-to-have).

(ii) The guidelines are those described in Section 3.1.
TABLE 3: Single element physical parameters.

\begin{tabular}{lcc}
\hline Parameter & Value & Note \\
\hline$\varepsilon_{r}$ & 2.2 & Dielectric constant \\
$t$ & 4 & Substrate thickness \\
$a$ & 2.8 & Patch arm width \\
$b$ & 2.8 & Patch arm length \\
$M_{x}$ & 6.1 & Mushroom width \\
$M_{y}$ & 6.1 & Mushroom length \\
mt & 0.1 & Mushroom thickness \\
dp & 1.8 & Probe plate diameter \\
$s$ & 0.15 & Gap probe-patch \\
Vd & 0.7 & Via diameter \\
Ph & 2 & Parallel plates capacitors height \\
dh & 3.85 & Parasitic element height $(t-s)$ \\
$g$ & 0.2 & Gap between metals \\
$o$ & 1.9 & Feed offset \\
$L_{x}$ & 18.4 & Square ring $x$-size \\
$L_{y}$ & 18.4 & Square ring $y$-size \\
H-port $x$ & 1.9 & Feed offset \\
H-port $y$ & 9.2 & $L_{y} / 2$ \\
V-port $x$ & 9.2 & $L_{x} / 2$ \\
$V$-port $y$ & 1.9 & Feed offset \\
\hline
\end{tabular}

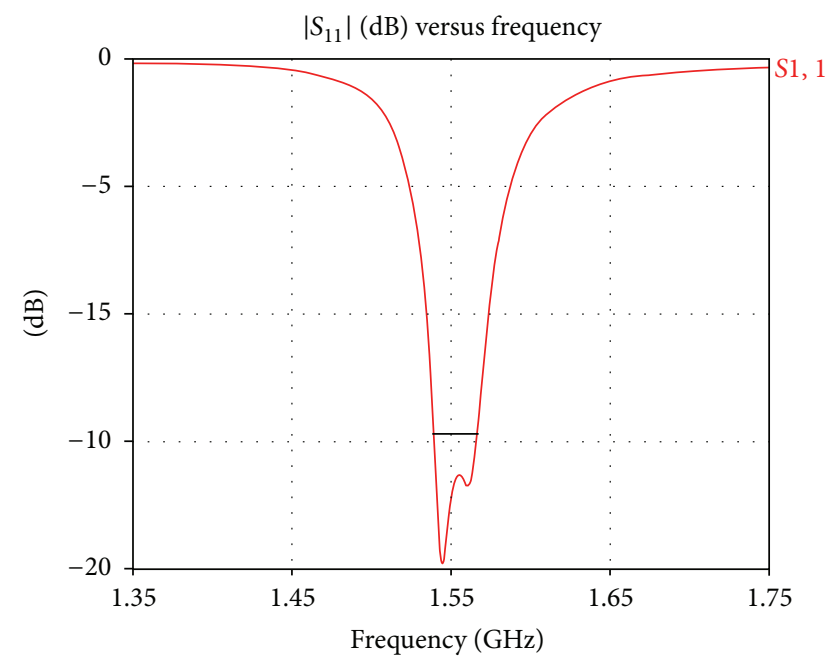

FIGURE 6: Single element return loss versus frequency.

Two layouts have been identified, that is, (i) the 13 elements, and (ii) the 7 elements configuration, as depicted in Figures 9 and 10, respectively. The first configuration is compliant to the $80 \mathrm{~cm}$ envelope constraint, while the second one to the nice-to-have constraint (i.e., $30 \mathrm{~cm}$ ). The geometry proposed in the 7 elements configuration has been already studied in the frame of GNSS studies [15]. Both layouts show physical symmetry along three symmetry axes, as depicted in Figures 9 and 10. As a consequence, a symmetrical pattern shall derive. It will optimize the GNSS satellites visibility. 
Gain (dBi) versus $\theta$ along $\Phi=0^{\circ}$ cut, $f=1.55 \mathrm{GHz}$

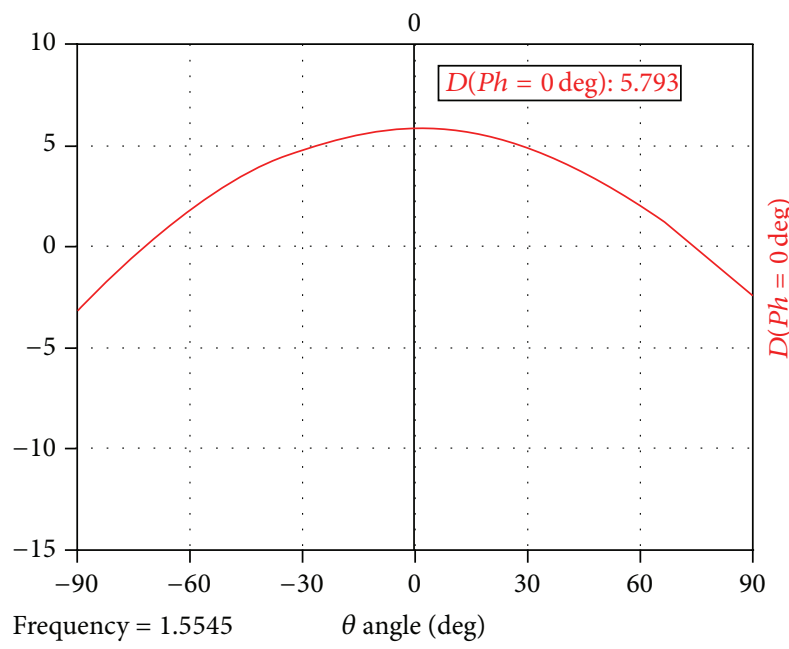

FIgURE 7: Single element gain versus elevation angle.

AR (dB) versus $\theta$ along $\Phi=0^{\circ}$ cut, $f=1.55 \mathrm{GHz}$

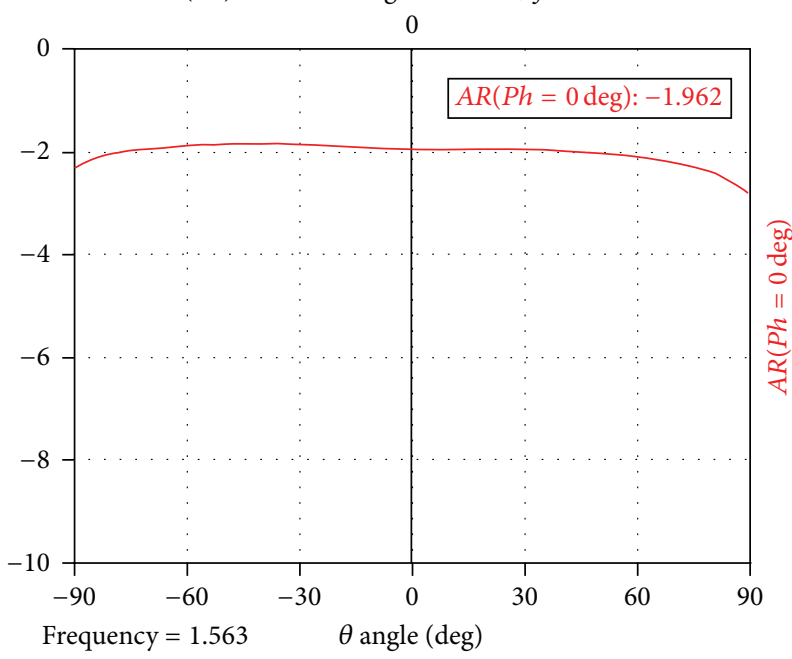

Figure 8: Single element axial ratio versus elevation angle.

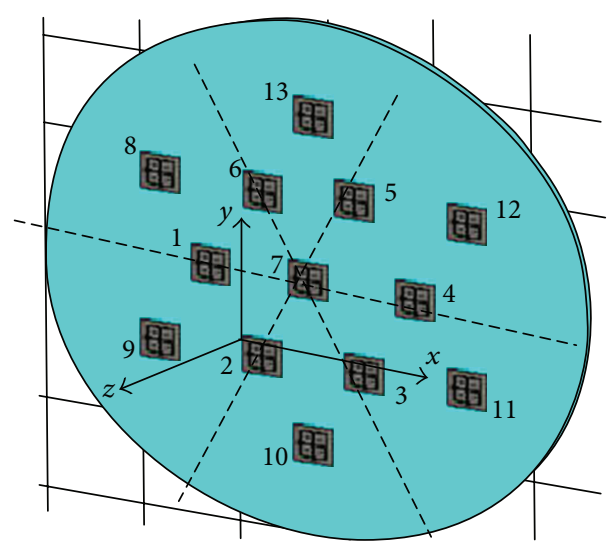

FIGURE 9: 13 elements array configuration-3D view.

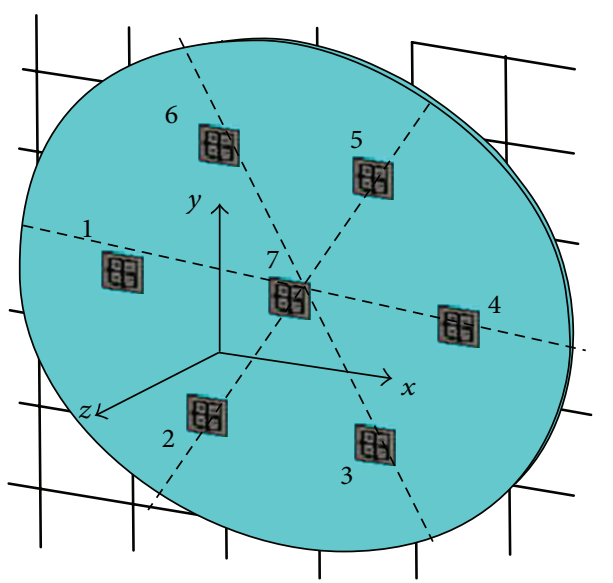

FIgURE 10: 7 elements array configuration-3D view.

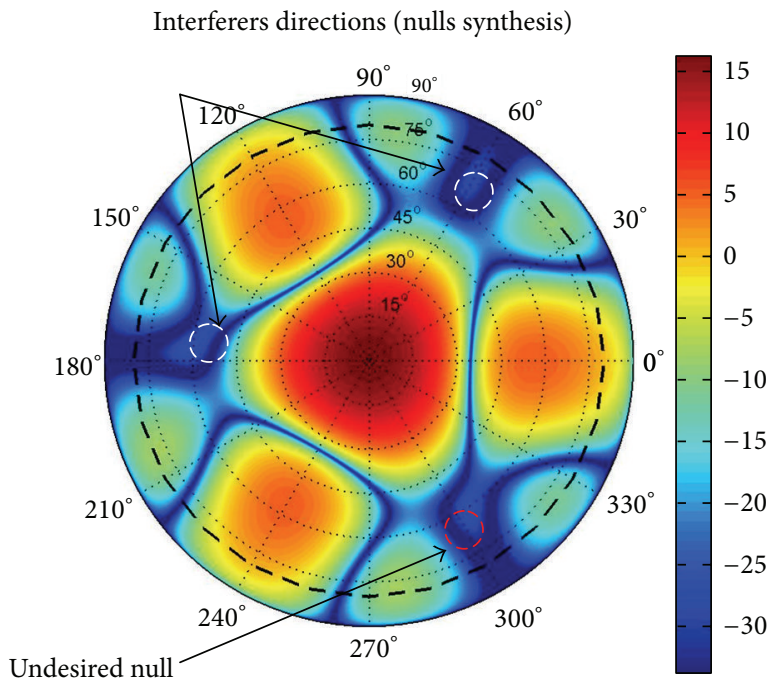

FIgURE 11: 7 elements array adaptive beam-forming antenna pattern-two nulls synthesis.

In both layouts the radiating elements are $0.73 \lambda_{g}$ spaced. This value derives from a trade-off between accommodation issues $\left(d / \lambda_{g}>0.5\right)$ and grating lobes effect $\left(d / \lambda_{g}<1\right)$.

Assuming $0.73 \lambda_{g}$, for the 7 elements configuration and radiator in metamaterial technology, the antenna diameter is $20.57 \mathrm{~cm}$. The total envelope is obtained by adding the $25 \%$ of antenna diameter value. The value is compliant to the accommodation constraint $(<30 \mathrm{~cm})$.

It is noted that considering standard antenna technology the overall envelope of the antenna would have exceeded the above-mentioned constraint.

The excitation coefficients for elements of the configurations at 13 and at 7 elements have been optimized by MVDR algorithm in order to synthesize nulls (up to 6) keeping the main lobe pointing $\theta=0^{\circ}$ (zenith direction).

Figure 11 shows an example of synthesis of two nulls for 7 elements antenna, along $\theta_{1}=60^{\circ}, \Phi_{1}=60^{\circ}$ and $\theta_{2}=70^{\circ}, \Phi_{2}=180^{\circ}$ directions. They are marked in white. On the other side, main beam-steering direction is set to 


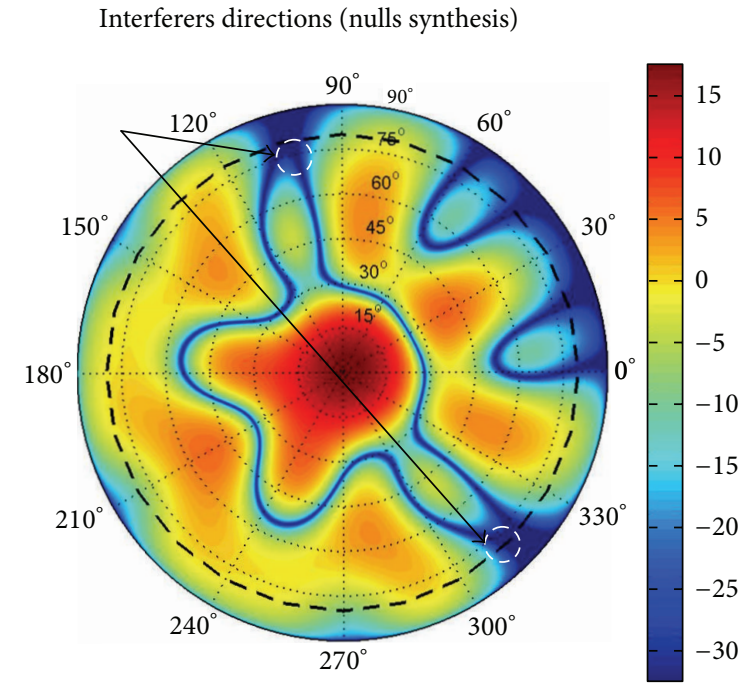

Figure 12: 13 elements array adaptive beam-forming antenna pattern-two nulls synthesis.

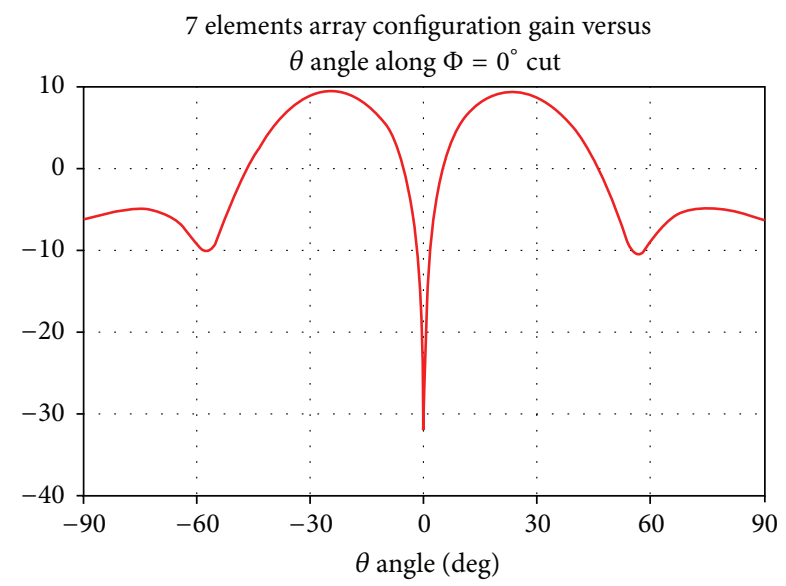

Figure 13: 7 elements array: adaptive beam-forming antenna pattern-single null synthesis.

TABLE 4: Excitation synthesized coefficients of pattern shown in Figure 13.

\begin{tabular}{lcc}
\hline Element Id. & Amplitude $[\mathrm{dB}]$ & Phase $[\mathrm{deg}]$ \\
\hline 1 & 0 & 0 \\
2 & 0 & +60 \\
3 & 0 & +120 \\
4 & 0 & +180 \\
5 & 0 & +240 \\
6 & 0 & +300 \\
7 & -30 & 0 \\
\hline
\end{tabular}

$\theta_{0}=0^{\circ}$ (zenith direction, $\Phi_{0}=0^{\circ}$ ). (Note that $\theta$ angle defined in antenna reference system (par. 3) and $\theta$ elevation angle defined in GNSS user reference system (par. 4) are $90^{\circ}$ offsets: zenith direction is $\theta=0^{\circ}$ at par. 3 , it is $\theta=90^{\circ}$

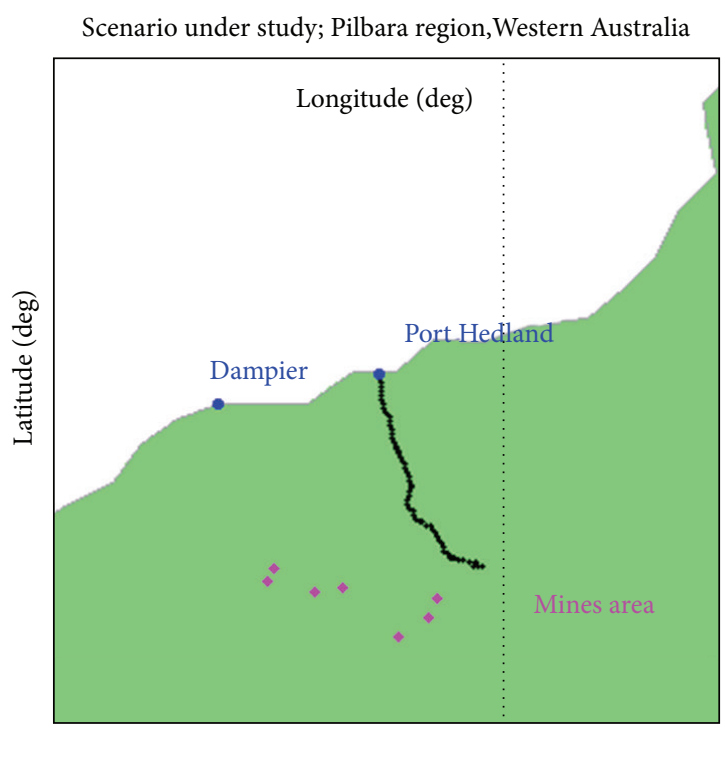

_ Number of user positions over the route: 2881

FIGURE 14: GNSS scenario under study—train path trajectory.

TABLE 5: 7 elements array: cases considered in the navigation realistic scenario.

\begin{tabular}{|c|c|}
\hline Case & Meaning \\
\hline FRPA cosine-like & $\begin{array}{l}\text { The radiation pattern of the user antenna } \\
\text { is a fixed beam approximated by a cosine } \\
\text { function ( } p \text { : integer). No interfering } \\
\text { satellite is considered in the analysis. }\end{array}$ \\
\hline FRPA simulated & $\begin{array}{l}\text { The radiation pattern of the user antenna } \\
\text { is a fixed beam (Figure 15) designed and } \\
\text { simulated by electromagnetic commercial } \\
\text { tool (i.e., "CST studio suite"). No } \\
\text { interfering satellite is considered in the } \\
\text { analysis. }\end{array}$ \\
\hline $\begin{array}{l}\text { FRPA cosine-like } \\
\text { with IF }\end{array}$ & $\begin{array}{l}\text { The radiation pattern of the user antenna } \\
\text { is a fixed beam approximated by a cosine } e^{p} \\
\text { function ( } p \text { : integer). Interfering satellite } \\
\text { is considered in the analysis. }\end{array}$ \\
\hline $\begin{array}{l}\text { FRPA simulated with } \\
\text { IF }\end{array}$ & $\begin{array}{l}\text { The radiation pattern of the user antenna } \\
\text { is a fixed beam (Figure 15) designed and } \\
\text { simulated by electromagnetic commercial } \\
\text { tool. Interfering satellite is considered in } \\
\text { the analysis. }\end{array}$ \\
\hline CRPA & $\begin{array}{l}\text { The radiation pattern of the user antenna } \\
\text { is synthesised by MVDR algorithm } \\
\text { (beam-forming) implemented by } \\
\text { "MatLab" software tool. In other words, } \\
\text { the antenna dynamically synthesizes } \\
\text { null/s along direction of interferer/s. } \\
\text { Interfering satellite is considered in the } \\
\text { analysis. }\end{array}$ \\
\hline
\end{tabular}

at par. 4.) It is noted that antenna diagram shows nulls also in noninterfering directions (e.g., at $\theta_{1}=70^{\circ}, \Phi_{1}=300^{\circ}$, marked in red). This can limit the visibility of GNSS (useful) satellites. The decrease of the number of tracked satellites can 


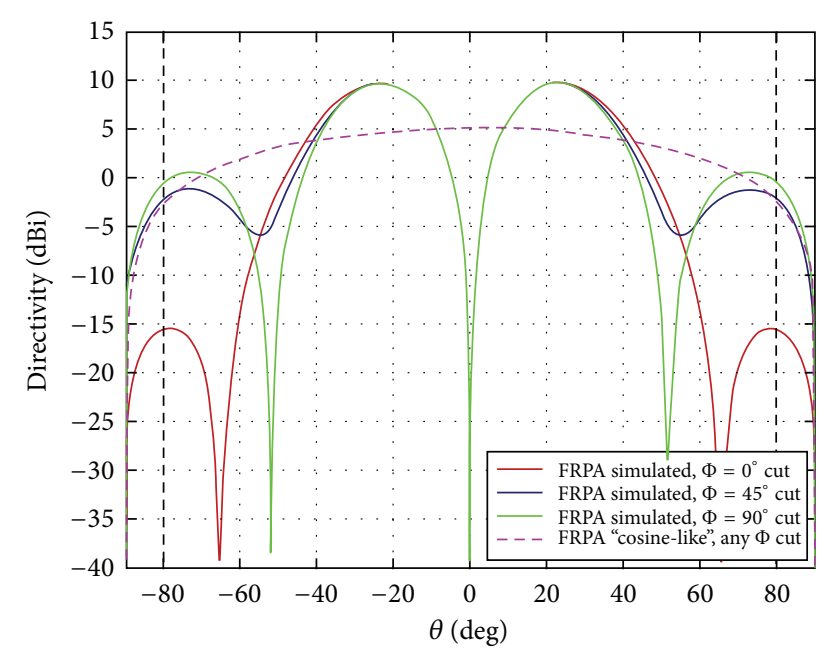

FIGURE 15: Ground planar array antenna. FRPA simulated and "cosine-like" pattern.

TABLE 6: UERE figure for GPS L1 receiver.

\begin{tabular}{lcc}
\hline It. & Error source & Value (rms-m) \\
\hline 1 & Satellite clock synchronization & $0^{1}$ \\
2 & Satellite perturbations & $0^{2}$ \\
3 & Ephemeris prediction error & $0^{1}$ \\
4 & Ionosphere delay & $4.87^{3}$ \\
5 & Troposphere delay & $0.1^{4}$ \\
6 & Multipath & $0.2^{5}$ \\
7 & Receiver noise and resolution thermal noise & $0.20^{6}$ \\
8 & UERE & $\mathbf{4 . 8 7}$ \\
\hline
\end{tabular}

${ }^{1}$ GNSS receiver augmented by SBAS satellites (e.g., QZSS for Australia) from direct signal in space (SIS).

${ }^{2}$ GNSS receiver augmented by SBAS satellites.

${ }^{3}$ Single frequency GNSS receiver, no corrections applied.

${ }^{4}$ Value obtained by "Ifadis" model [5].

${ }^{5}$ Assuming signal-to-multipath ratio: $27.5 \mathrm{~dB}$, path delay: $26 \mathrm{~m}$, E-L narrow spacing: 0.1 chip, signal bandwidth $=24 \mathrm{MHz}$.

${ }^{6}$ Assuming noncoherent discriminator DLL with early-late spacing: 0.1; C/No: $40 \mathrm{~dB}-\mathrm{Hz}$; code loop noise bandwidth: $0.1 \mathrm{~Hz}$ integration time: $20 \mathrm{msec}$; chip length of code: $293.2551 \mathrm{~m}$.

lead to a reduction of the service accuracy/time availability. This impact shall be evaluated in the next paragraph in the frame of the study of the navigation realistic scenario.

The 13 elements configuration design does not meet the $30 \mathrm{~cm}$ constraint. It is considered as a back-up design. However, as a title of example, Figure 12 shows a radiation pattern for a 13 elements array configuration in case of two nulls synthesis.

Phased array electromagnetic analysis has been addressed by electromagnetic commercial tool (i.e., CST Studio Suite) in order to take into account RF mutual coupling effects: in Figure 13, another example of radiation pattern optimization is provided. It represents a null synthesis along broad-side (zenith) direction. The diagram appears balanced, symmetrical with respect to zenith direction. The antenna

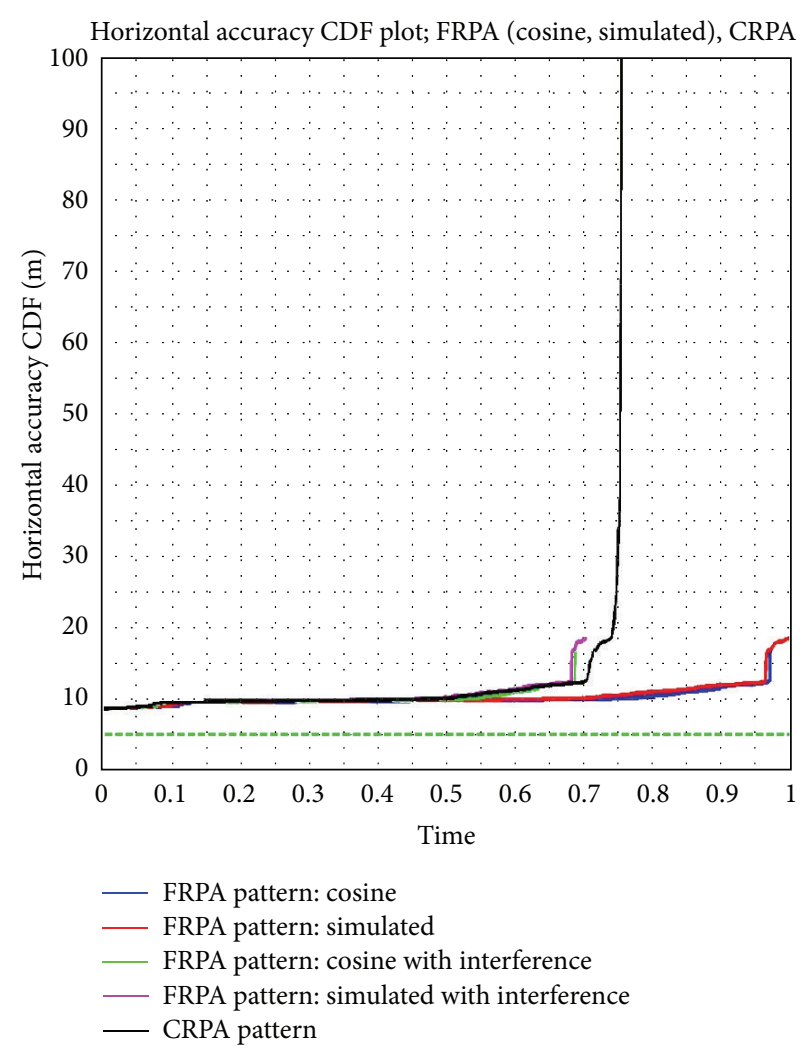

FIGURE 16: Horizontal accuracy CDF curve -24/24 GPS constellation and $1 \mathrm{RF}$ interfering satellite.

RF design allows the rejection to the interference signal (supposed along zenith direction in this case) and, on the other side, the maximization of the gain in order to receive the maximum number of GNSS useful signals with a signalto-noise ratio adequate to ensure the tracking of the GNSS receiver [16].

It is important to note that the symmetry of the radiation pattern and the depth of the null (about $-30 \mathrm{~dB}$ in Figures 11 and 13) demonstrate that degradation effects like RF coupling among elements and beam-forming network effects (i.e., excitation coefficients truncation) are well taken into account in the antenna RF design.

In the current paper, focus is given to the innovative radiating part of the antenna. Contribution of the antenna feeding network (i.e., typical values of excitation coefficients stability over frequency/temperature, truncation of excitation coefficients) is taken into account in the design.

The excitation synthesized coefficients are shown in the following Table 4 (see also Figure 10).

Finally, it is worth to note that antenna centre of phase has not been considered due to negligible contribution to accuracy error (order of millimetres).

\section{GNSS Study Case}

In the current paragraph, the design of the phased array shown in the previous part is applied to a GNSS user receiver 


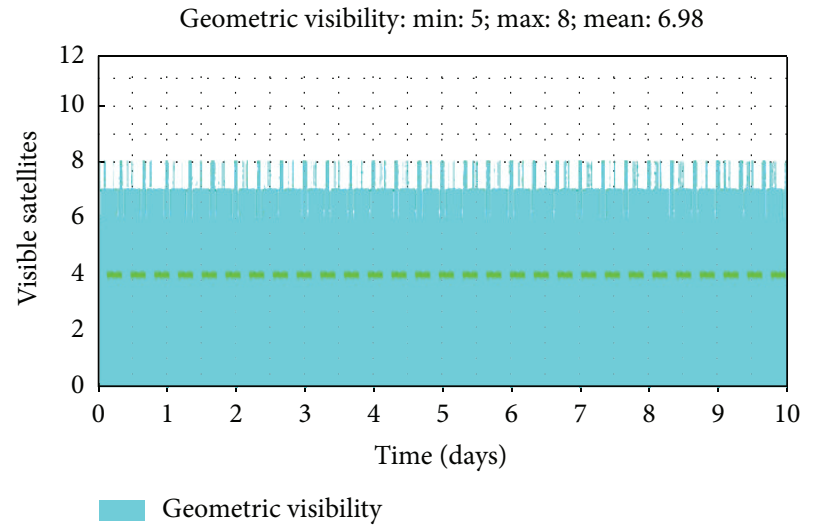

(a)

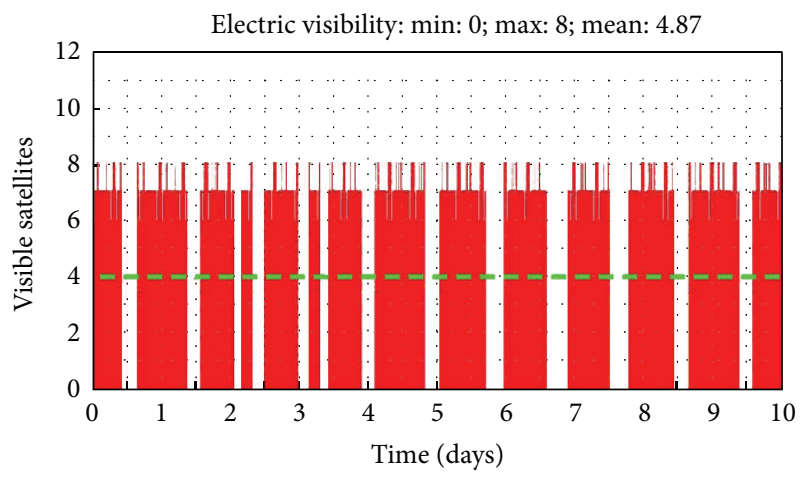

Electric visibility

(b)

FIGURE 17: Number of visible satellites -24/24 GPS constellation and $1 \mathrm{RF}$ interfering satellite.

antenna in a navigation realistic environment consisting of

(i) GNSS satellite constellation (e.g., GPS, Galileo, GLONASS, and Compass);

(ii) SBAS satellite constellation (e.g., QZSS for Japan and Australia, EGNOS for Europe, and WAAS for U.S.);

(iii) GNSS user receiver antenna accommodated on the train roof. The train is travelling along a more than $200 \mathrm{~km}$ long route from a mines area to "Port Headland" (and back) in Western Australia region, rural rail environment at an average speed of $80 \mathrm{~km} / \mathrm{h}$ (Figure 14);

(iv) Satellite constellation of interfering satellites (from 1 to 6 , randomly selected) transmitting $20 \mathrm{dBW}$ over the GPS EIRP nominal value on L1 bandwidth;

(v) Train running in adverse conditions (totally disconnected scenario, no ground infrastructures).

In particular, the GNSS antenna (7 elements array discussed at par. 3.1, 3.2) is supposed to work in FRPA or CRPA modes. Consequently, five cases are taken into account (Table 5).

Figure 15 shows the FRPA simulated pattern (three $\Phi$ cuts) and the FRPA "cosine-like" pattern (pink dotted line).

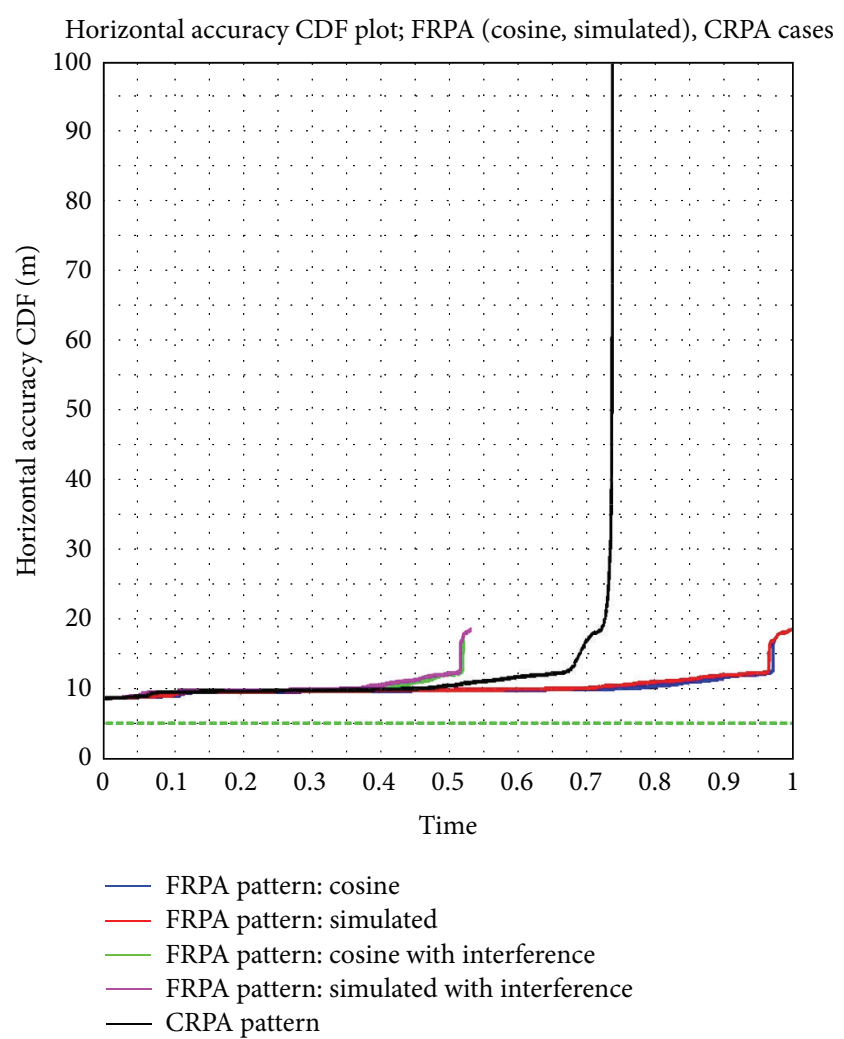

Figure 18: Horizontal accuracy CDF curve -24/24 GPS constellation and $2 \mathrm{RF}$ interfering satellites.

The assumptions followed in the realistic scenario simulation are the following.

(i) The observation period is 10 days, 5 minutes sampling: the train is running from mines area point to "Port Headland" and back; the total number of points described by the trajectory is 2881 ;

(ii) the accuracy value is computed with $95 \%$ confidence level;

(iii) GPS signal on-ground power level as defined in [16];

(iv) the attenuation by atmospheric gases is computed by ITU-R P.676-2 model [17];

(v) the rainfall attenuation is modelled as described in [17];

(vi) masked areas as those pertaining to a tunnel and bridges are neglected;

(vii) no GNSS satellite failure (i.e., on-board clocks) is assumed;

(viii) GNSS receiver mask angle $>10^{\circ}$ with respect to horizon (as shown in Figure 15);

(ix) GPS receiver acquisition threshold: $30 \mathrm{~dB}-\mathrm{Hz}$ (typical value);

(x) GNSS user receiver antenna given by a 7 elements phased array working in FRPA mode (cosine-like pattern or simulated in Figure 15) or CRPA mode; 


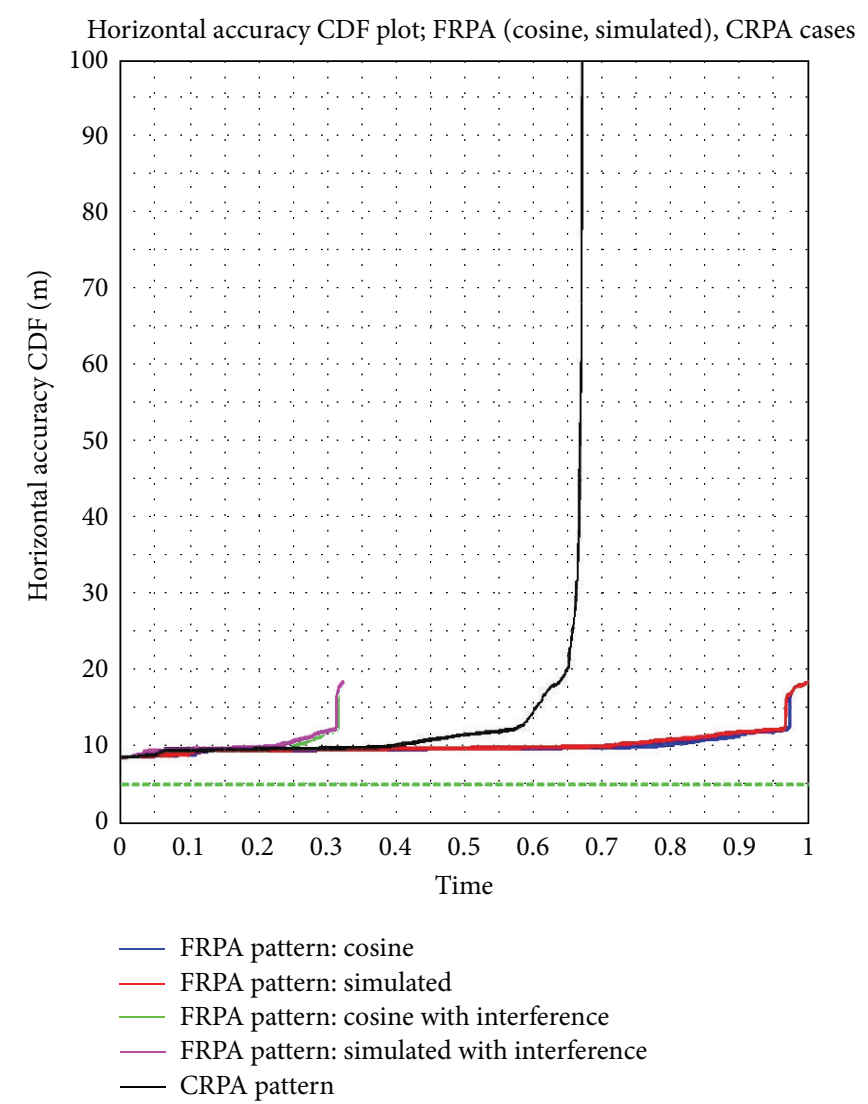

FIGURE 19: Horizontal accuracy CDF curve -24/24 GPS constellation and $3 \mathrm{RF}$ interfering satellites.

(xi) user equivalent ranging error (UERE) figure is considered common to each GPS satellite. The value is equal to 4.87 meters (at $1 \sigma$ ) computed at $\theta=$ $15^{\circ}$ (conservative approach). Source error typical standard deviations in L1 band only are reported in Table 6.

The UERE budget driver is the ionosphere residual error.

For each case of the described navigation scenario, user location 2D accuracy versus time and service availability figures are computed, and the results are compared in order to have evidence of the impact of user antenna pattern on the GNSS service performance.

In particular, a constellation of 24/24 GPS satellites disposed over 6 orbital planes (4 satellites per plane) is studied. RF interferers are gradually introduced (from 1 to 6) in the scenario. Figures 16-24 show the horizontal accuracy cumulative distribution function (CDF) for the cases described in Table 5.

In Figure 16, in absence of interference, the horizontal accuracy at a time availability of $95 \%$ is about 12 meters both for FRPA cosine and simulated pattern antenna. In interference conditions, the GPS receiver "sees" a useful link with the GPS satellite, whose $C / N_{0}$ is between 35 and $50 \mathrm{~dB}$ $\mathrm{Hz}$ (according to user elevation angle) and noise contribution $I_{0}$ due to RF interference.

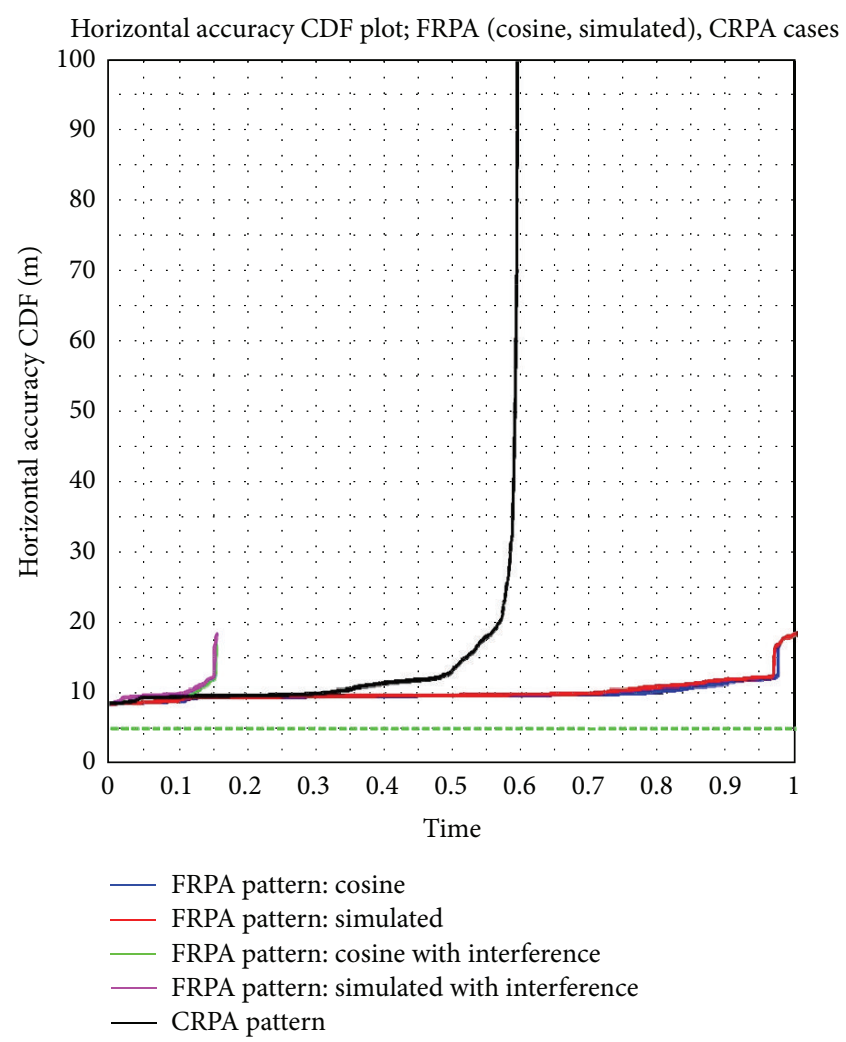

FIGURE 20: Horizontal accuracy CDF curve -24/24 GPS constellation and $4 \mathrm{RF}$ interfering satellites.

Consequently, total GPS satellite $C /\left(N_{0}+I_{0}\right)$ figure is degraded; the signal-to-noise ratio starts to decrease as soon as the interfering satellite is in visibility of the GPS user receiver. If the GPS satellite signal-to-noise ratio is less than the receiver acquisition threshold, satellite signal cannot be acquired and satellite cannot be tracked by the receiver, so the number of useful (necessary to compute user position) satellites starts to decrease.

This concept is illustrated in Figure 17. It represents the number of GNSS satellites geometrically visible (in light blue), electrically visible (in red) to the train along its route (versus observation time). In absence of interference, satellites that are visible geometrically are also electrically (even with a $0 \mathrm{dBi}$ gain antenna) visible. In case of interference, as mentioned before, the useful signal power shall be below the noise (thermal and interference nature) more than $30 \mathrm{~dB}-\mathrm{Hz}$ leading to the loss of satellite track. In addition, the $\mathrm{DOC}_{4}$ figure (Depth of Coverage 4: time percentage to have at least four satellites in visibility to the GPS receiver) shall pass from $100 \%$ to $70.53 \%$ as shown in Figure 16 . (Note that 4 is the minimum number of GNSS satellite to compute the user 3D location.) The threshold of 4 satellites (minimum number to compute GNSS user position) is highlighted in Figure 17 by dotted green line.

Consequently, the user accuracy is computed by a reduced number of satellites. It is degraded (about 18 meters) with a reduced availability (about 70\%) both for cosine and simulated FRPA antennas. Adopting the CRPA solution, 
Horizontal accuracy map; FRPA: cosine-like pattern in IF conditions min: 8.63 m; max: $18.38 \mathrm{~m}$; time availability: $15.89 \%$

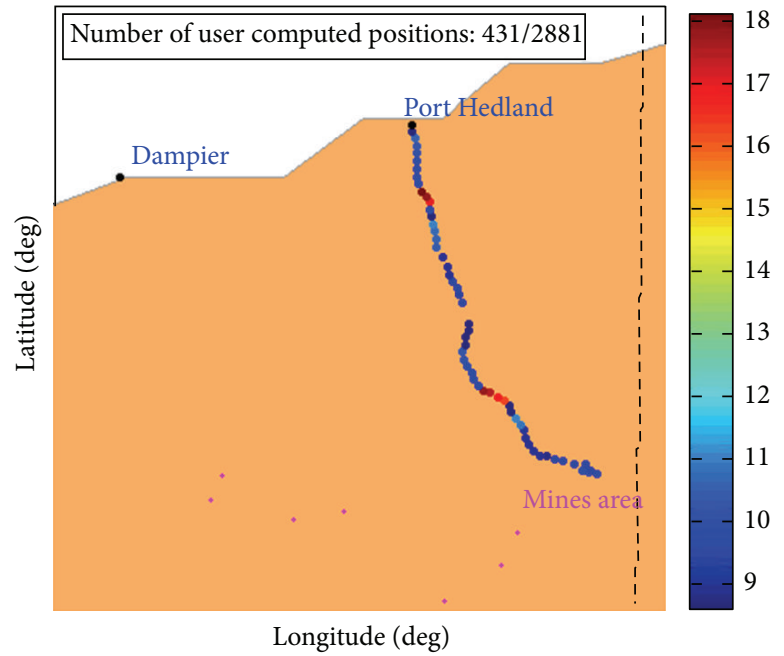

FIGURE 21: Horizontal accuracy mapping -24/24 GPS constellation and $4 \mathrm{RF}$ interfering satellites.

a null is synthesized along interfering direction and the antenna main beam points the broad-side direction. It mitigates the effects of the interference; however, the synthesized radiation pattern can show undesired nulls that can limit the GPS satellite visibility (Figure 11). Consequently, the service can be provided with a maximum $2 \mathrm{D}$ accuracy of 30 meters with a time availability of about $75 \%$.

In Figure 18 two interfering satellites are considered. The $2 \mathrm{D}$ accuracy figure is reduced to $18 \mathrm{~m}$ with $52 \%$ time availability both for FRPA cosine and FRPA simulated. CRPA antenna solution mitigates the interference effect (30 meters accuracy with about $74 \%$ time availability). This value is very similar to the one obtained for one single interfering satellite case. It is noted that CRPA provides robustness to interference without affecting the GPS satellite visibility.

For the 3 interfering satellites option (Figure 19), the horizontal accuracy of the train is degraded to 18 meters at $52 \%$ of the time for FRPA patterns. Adopting CRPA solution it is 30 meters at $68 \%$ of the time (10 days).

On each curve, a reference value of 5 meters has been considered (green dotted line). An horizontal accuracy of 5 meters represents a typical value for train applications (this is why user antenna phase centre error contribution is not taken into account). This value can be even lower when additional capabilities, like parallel track discrimination is required [18].

The multiconstellation capabilities relying on GPS, GLONASS, and in perspective Galileo and Compass offer a higher degree of flexibility to reach better performance.

For the 4 interfering satellites option (Figures 20 and 21), the horizontal accuracy is degraded to 18 meters at about $15 \%$ of the observation time for FRPA patterns. Adopting CRPA solution it is 30 meters at $55 \%$ of the time.

More specifically, it means that, in conditions of interference, the train position can be computed by the GPS receiver in $15 \%$ of the total time (in other words, for the $15 \%$ of the

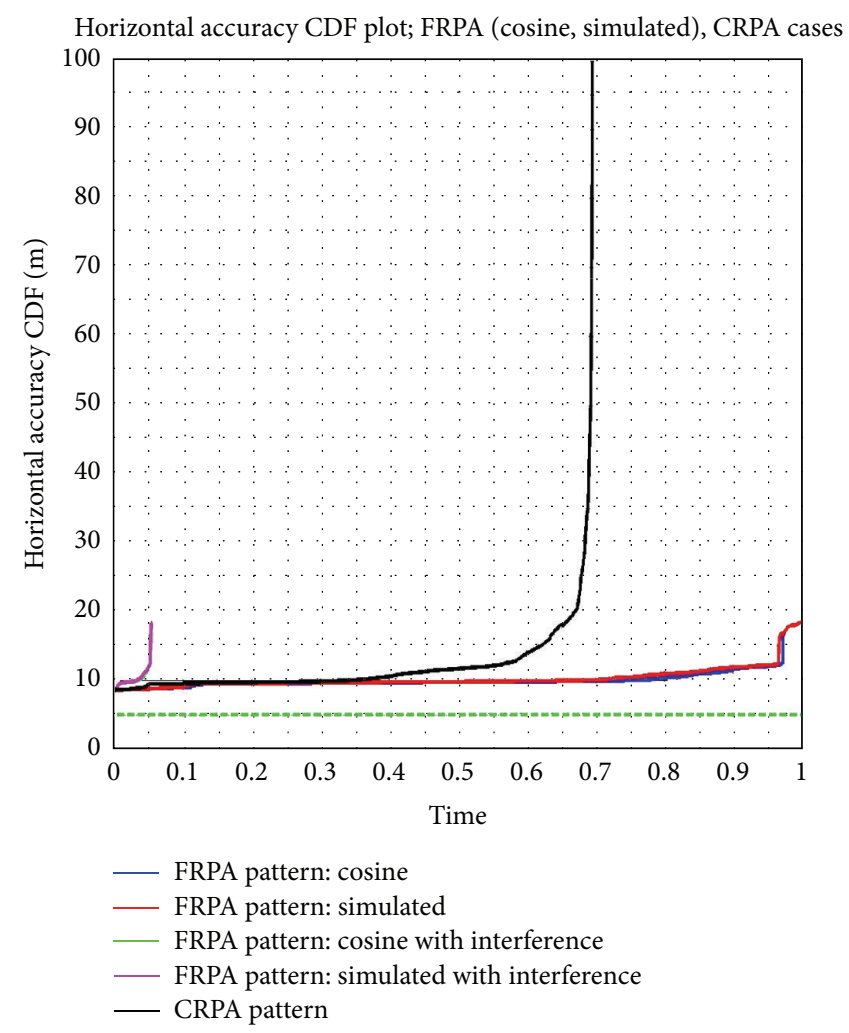

FIgURE 22: Horizontal accuracy CDF curve -24/24 GPS constellation and 5 RF interfering satellites.

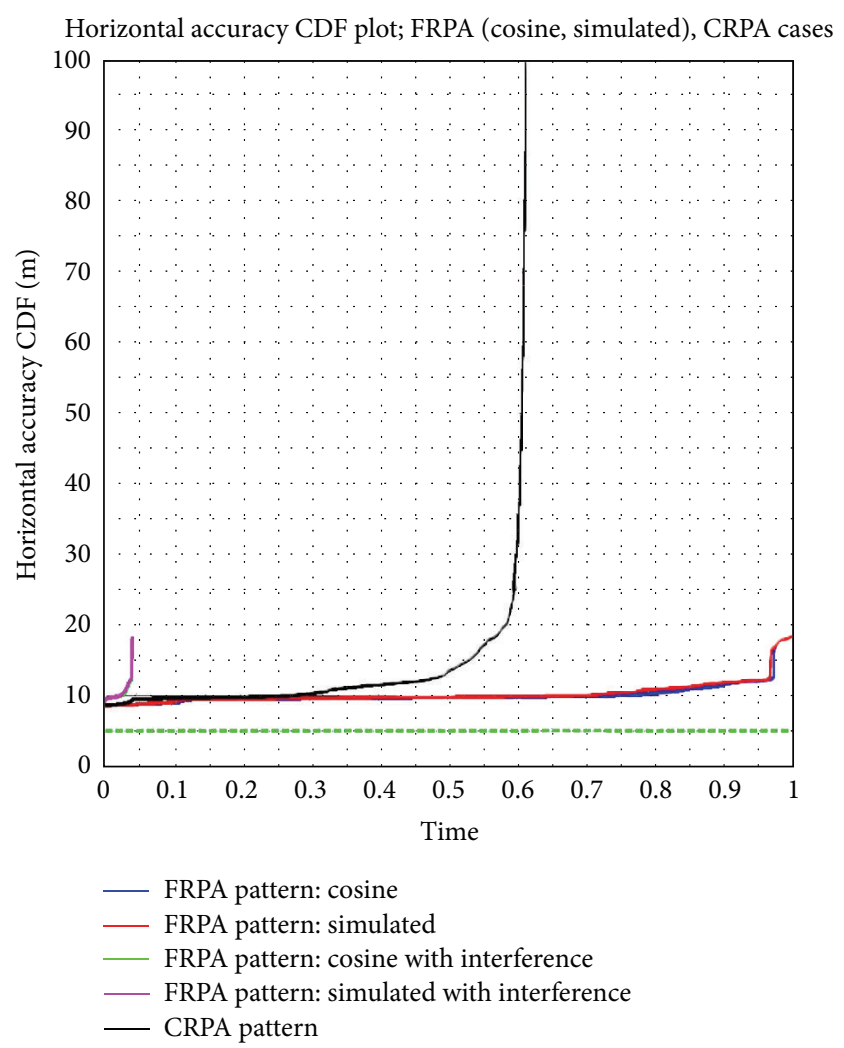

FIGURE 23: Horizontal accuracy CDF curve -24/24 GPS constellation and $6 \mathrm{RF}$ interfering satellites. 
Horizontal accuracy map; FRPA: cosine-like pattern in IF conditions min: $8.65 \mathrm{~m}$; max: $18.3 \mathrm{~m}$; time availability: $4.44 \%$

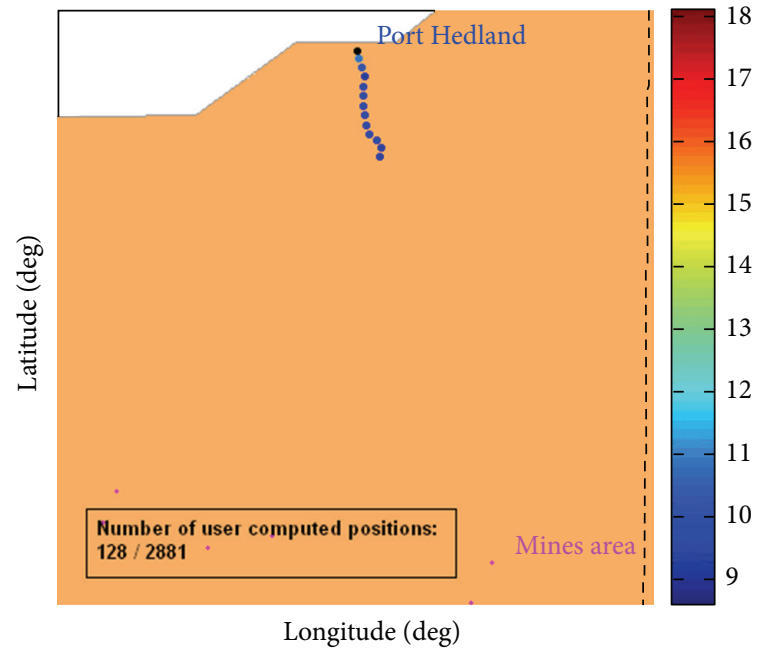

FIGURE 24: Horizontal accuracy mapping ("zoomed" view) $-24 / 24$ GPS constellation and 6 RF interfering satellites.

points of the train route shown at Figure 14) with a minimum $2 \mathrm{D}$ accuracy of $8.63 \mathrm{~m}$ and a maximum 2D accuracy of $18.38 \mathrm{~m}$ (Figure 21).

For the 5 interfering satellites option (Figure 22), the horizontal accuracy is degraded to 18 meters at $5 \%$ of the time for FRPA patterns. Adopting CRPA solution it is 30 meters at $68 \%$ of the time (10 days).

For the 6 interfering satellites option (Figures 23 and 24), the horizontal accuracy is degraded to 18 meters at $5 \%$ of the time for FRPA patterns. It is noted that CRPA antenna solution strongly mitigates the interference effects. In other words, the train position can be located along the route with a $2 \mathrm{D}$ maximum accuracy of 30 meters at $60 \%$ of the time $(10$ days).

By adopting an FRPA antenna, the 2D position of the train is known with an accuracy of 18.3 meters (Figure 23) at about the $5 \%$ of the train route (Figure 14). Comparing Figures 24 and 13 , it is noted that the points (user positions computed by the receiver-128) do not encompass the whole rail path (2881 points). It is due to the lack of GPS visible satellites (caused by RF interference) to the receiver. If the number of GPS satellites visible is less than 4 , the receiver cannot compute the user position; consequently, the marker is, respectively, blank in Figures 21 and 24.

\section{Conclusions}

In this paper an innovative radiating system for train localization in interference conditions is presented. This system consists of a beam-forming adaptive antenna, a phased array of miniaturized (metamaterial technology) patch radiators. The design of the beam-forming antenna is presented (working in FRPA or CRPA mode).

Phased array antenna is embedded into a navigation realistic scenario in interference conditions, and the problem of localization-by-GNSS-satellite of a train travelling along a route in Australia is approached.

Service performance figures (i.e., accuracy, time availability) are evaluated, and emphasis is given to the capability of the GNSS-designed array to mitigate RF interference effects (reduction of service availability, increase of location accuracy).

\section{References}

[1] M. Jones, "Protecting GNSS receivers from interference and jamming," Inside GNSS, pp. 40-49, March-April 2011.

[2] E. D. Kaplan and C. J. Hegarty, Understanding GPS: Principles and Applications, Artech House, 2006.

[3] A. Joseph, "Measuring GNSS signal strength," Inside GNSS, pp. 20-25, November-December 2010.

[4] H. L. Van Trees, Optimum Array Processing, Part IV of Detection, Estimation, and Modulation Theory, John Wiley \& Sons, 2002.

[5] S. Katsougiannopoulos, C. Pikridas, D. Rossikopoulos et al., "Tropospheric refraction estimation using various models, radiosonde measurements and permanent GPS data," in Shaping the Change, XXIII FIG Congress, Munich, Germany, October 2006.

[6] R. T. Campton Jr., Adaptive Antennas: Concepts and Performance, Prentice-Hall, 1988.

[7] F. Capolino, Ed., Metamaterials Handbook, CRC Press, 2009.

[8] C. Vegni and F. Bilotti, "Parametric analysis of slot-loaded trapezoidal patch antennas," IEEE Transactions on Antennas and Propagation, vol. 50, no. 9, pp. 1291-1298, 2002.

[9] S. Contu, C. Vegni, F. Bilotti, and L. Vegni, "Slotted patch antennas for space applications," in Proceedings of the USNC/URSI National Radio Science Meeting, p. 695, Columbus, Ohio, USA, June 2003.

[10] F. Bilotti and C. Vegni, "Design of metamaterial based patch antennas with reduced back-radiation for radio navigation systems," in Proceedings of the 1st International Congress on Advanced Electromagnetic Materials in Microwaves and Optics (Metamaterials '07), pp. 493-494, 2007, Rome, Italy, October 2007.

[11] F. Bilotti and C. Vegni, "Employment of artificial magnetic metamaterials to effectively reduce the back-lobe of patch antennas," Electromagnetics, vol. 28, no. 7, pp. 513-522, 2008.

[12] F. Bilotti and C. Vegni, "Design of high-performing microstrip receiving GPS antennas with multiple feeds," IEEE Antennas and Wireless Propagation Letters, vol. 9, pp. 248-251, 2010.

[13] G. Bertin, B. Piovano, R. Vallauri, F. Bilotti, and L. Vegni, "Metamaterial-inspired antennas for telecommunication applications," in Proceedings of the 6th European Conference on Antennas and Propagation (EuCAP '12), pp. 2739-2740, Prague, Czech Republik, March 2012.

[14] G. Bertin, F. Bilotti, B. Piovano, R. Vallauri, and L. Vegni, "Switched beam antenna employing metamaterial-inspired radiators," IEEE Transactions on Antennas and Propagation, vol. 60, no. 8, pp. 3583-3593, 2012.

[15] C. Vegni and F. Bilotti, "Spaceborne orbito payload study and L2c antenna design for precise geosynchronous orbit/time determination," in Proceedings of the European Navigation Conference-Global Navigation Satellite Systems (ENC-GNSS '09), Napoli, Italy, May 2009. 
[16] Global Positioning System Wing (GPS)-Systems Engineering \& Integration Interface Specification IS-GPS-200, Revision E. ISGPS-200E, June 2010.

[17] G. Maral and M. Bousquet, Satellite Communication Systems, Wiley, 5th edition, 2009.

[18] A. Neri, A. Filip, F. Rispoli, and A. M. Vegni, "An analytical evaluation for hazardous failure rate in a satellite-based train positioning system with reference to the ERTMS train control systems," in Proceedings of the 25th International Technical Meeting of the Satellite Division of the Institute of Navigation (ION GNSS '12), p. 2770, Nashville, Tenn, USA, September 2012. 

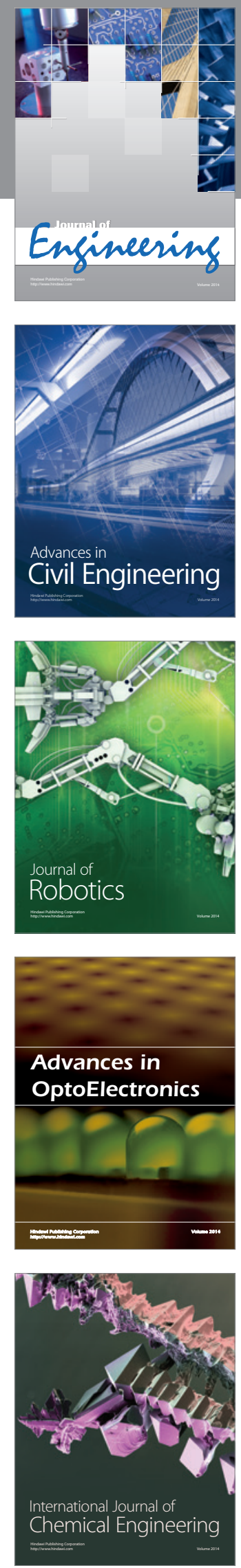

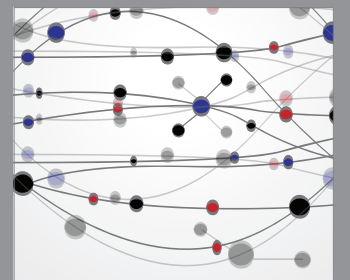

The Scientific World Journal
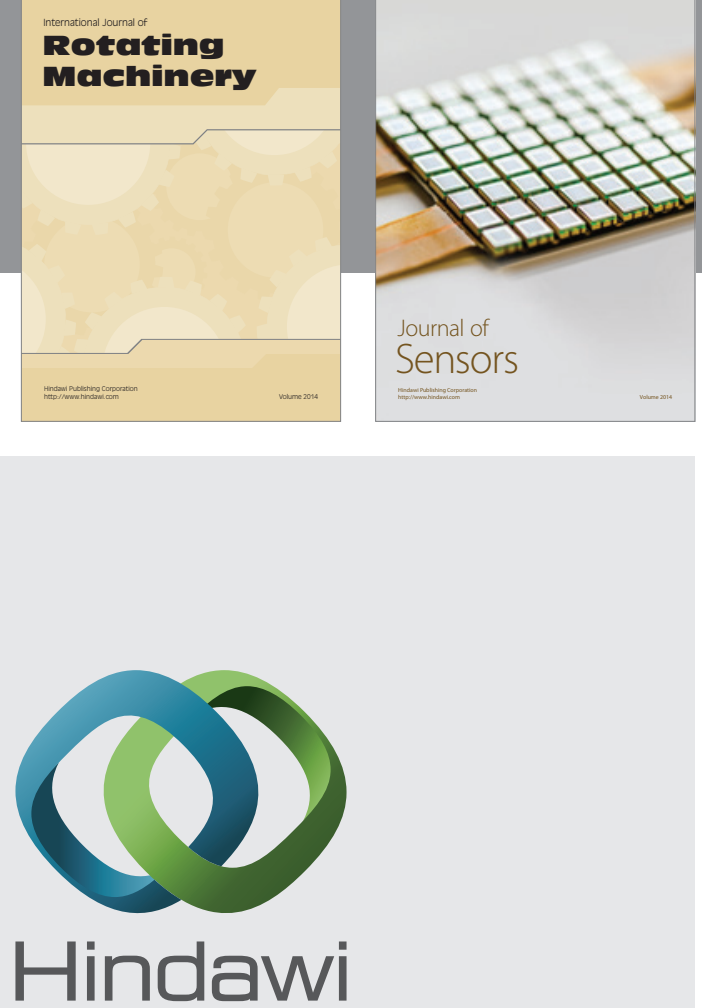

Submit your manuscripts at http://www.hindawi.com
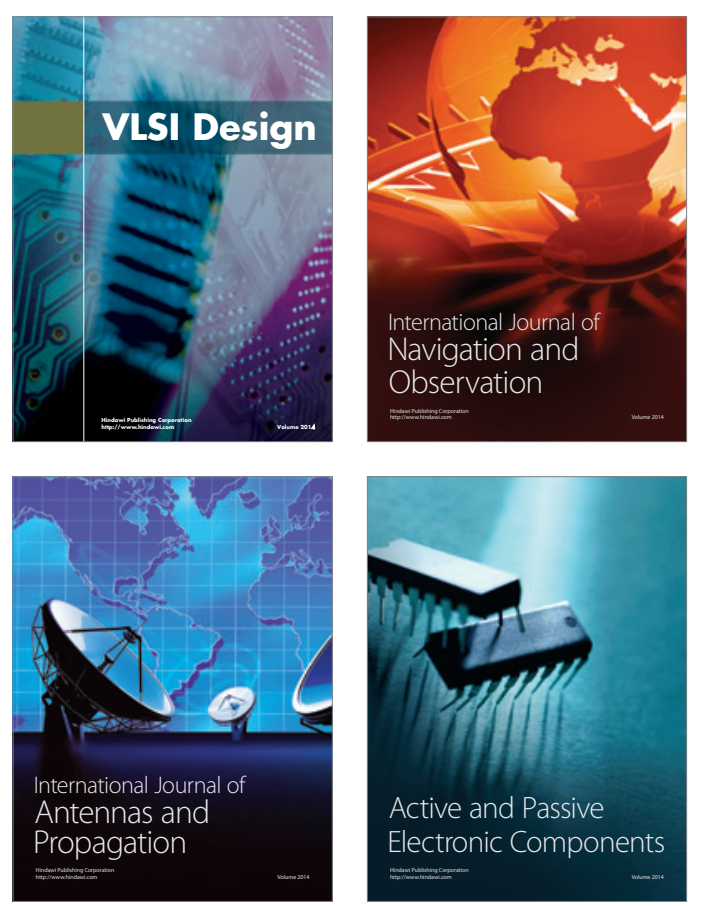
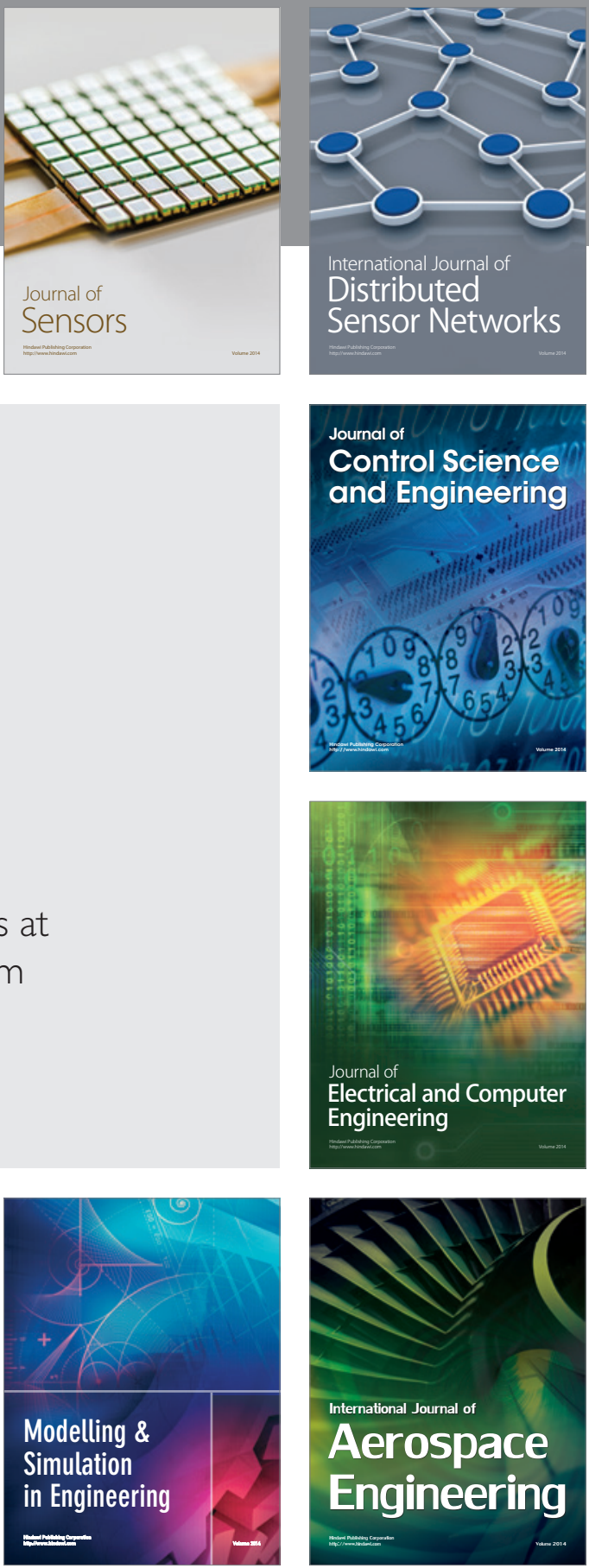

Journal of

Control Science

and Engineering
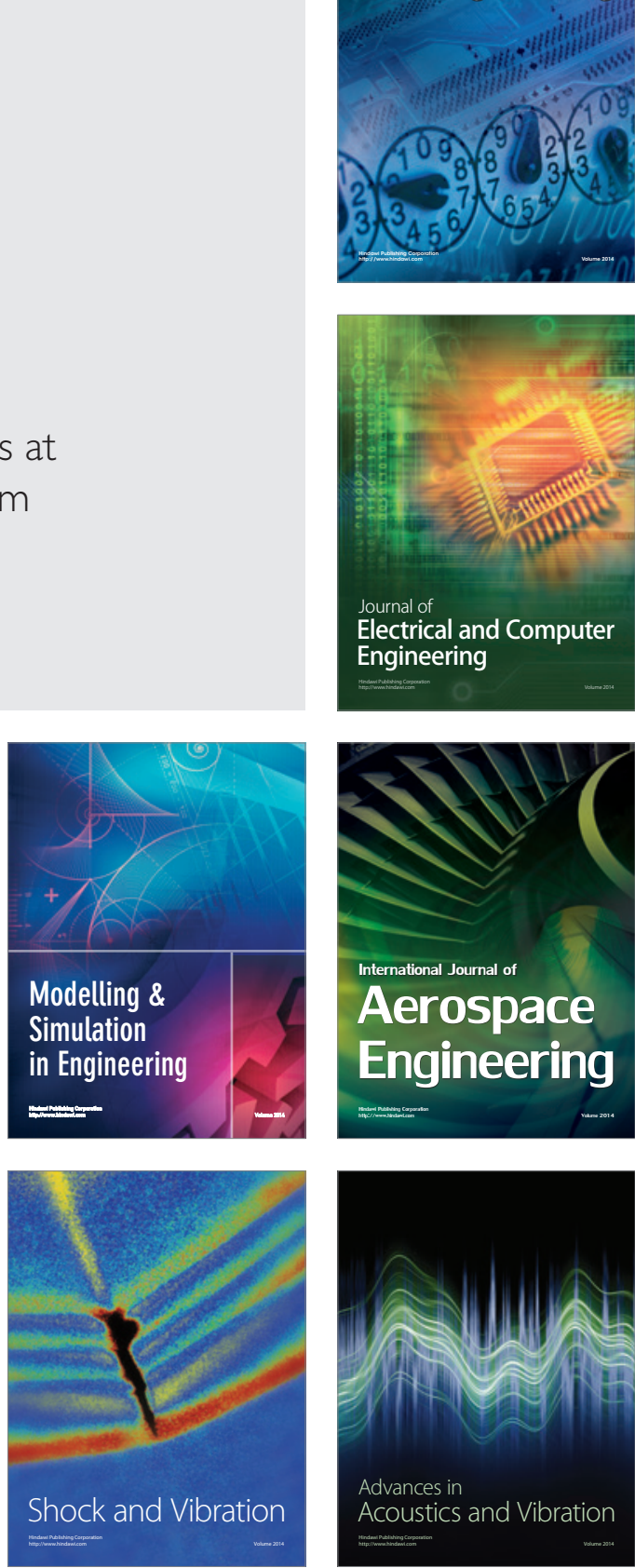